\title{
Protective effect of high concentration of BN52021 on retinal contusion in cat eyes
}

\author{
Jin-Feng Huang ${ }^{1}$, Hai-Peng Zhao ${ }^{2}$, Yan-Feng Yang ${ }^{1}$, Hui-Min Huang ${ }^{1}$, Yi Yao $^{3^{*}}$ and Zhi-Jun Wang ${ }^{2^{*}}$
}

\begin{abstract}
Background: Blunt injuries/contusion on eyes might cause retina blunt trauma. This study is to evaluate the protective function of BN52021 against retinal trauma.

Methods: A total of 70 cats, 6 months old, were divided into six groups: Group A to $E(n=12)$ and normal control (N) group $(n=10)$. The right eyes in Group A to E were contused. All experiments were performed under general anesthetization. Retrobulbar injections of medication in right eyes were performed. Cats were administrated with $0.5 \mathrm{~mL}$ of normal saline (NS), dimethyl sulphoxide, $0.2 \mathrm{~g} / \mathrm{L}$ BN52021, $1 \mathrm{~g} / \mathrm{L}$ BN52021 and $5 \mathrm{~g} / \mathrm{L}$ BN52021, respectively. Cats in Group N were administrated with $0.5 \mathrm{~mL}$ of NS. Intraocular pressure (IOP), flash electroretinogram (ERG), and retinal nerve fiber layer (RNFL) thickness were measured. Hematoxylin and eosin (HE) staining and transmission electron microscope (TEM) were detected.

Results: No significant difference was observed in IOP levels among groups. Comparing with cats in Group N, those in Group A to E showed significant lower amplitudes of rod a- and b-waves $(P<0.05)$. Amplitudes of rod $a$ - and b-waves were increased by administration of high concentration of BN52021 ( $\geq 1 \mathrm{~g} / \mathrm{L}$ ). Moreover, high concentration of BN52021 decreased the RNFL thickness increased by contusion. Axons in RNFL in Group E arranged neatly at 7 days after modeling.
\end{abstract}

Conclusions: The degenerated axons caused by contusion were repaired by BN52021. The administration of high concentration of ( $\geq 1 \mathrm{~g} / \mathrm{L})$ BN52021 could partially repair retinal function in contused cat eyes.

Keywords: Retina trauma, BN52021, Electroretinogram, Intraocular pressure, Retinal nerve fiber layer

\section{Background}

Contusion/superficial injuries (abrasion) is the most common cause for retina blunt trauma and most of the injuries (accounting for $>25 \%$ ) are closed globe contusion $[1,2]$. Blunt injury to eye results in both direct damage at the site of impact tissue and indirect damage to distant intraocular tissues by transmitted forces [3].

Ocular variables as intraocular pressure (IOP) $[4,5]$, electroretinogram (ERG) parameters [6], and retinal nerve fiber layer (RNFL) thickness $[7,8]$ are affected by retina blunt trauma. For instance, Nakayama et al. had reported that the amplitudes of the focal macular ERGs after retinal hemorrhages were lower than those of the

\footnotetext{
*Correspondence: yiyaodoubley@163.com; zhijunwangzjw@163.com ${ }^{3}$ Department of Ophthalmology, The General Hospital of PLA, No. 28 Fuxing Road, Beijing 100853, China

${ }^{2}$ Department of Ophthalmology, CHINA-JAPAN Friendship Hospital, No.2 Yinghua Dongjie, Hepingli, Beijing 100029, China

Full list of author information is available at the end of the article
}

full-field ERGs [6]. Ahn et al. evaluated RNFL thickness using optical coherence tomography (OCT). They determined that RNFL thickness at the second week after trauma was significantly higher than that at the 24th week [7].

Ginkgo biloba, which is commonly known as maidenhair tree, is available as a popular herbal supplementary in Asian, European and American countries. Evidences have showed that Ginkgolide B (BN52021, Ginkgo biloba extract) has protective function against retinal injuries $[9,10]$. BN52021 is an antagonist to platelet activating factor (PAF). BN52021 induces irreversible decrease of ERG parameters [9,11,12]. The administration of BN52021 could partially inhibit the irreversible decrease of ERG parameters induced by PAF $[9,11]$. The protective function of BN52021 on retina has not been systematically reported in cats up to now. 
Although there are various differences between human and cat eyes, cats have been serving as models for human vision for a long time [13]. In order to evaluate BN52021's protective function against retinal blunt trauma, cat models with contused eyes have been established in our study. BN52021 at different concentrations will be administrated to experimental groups. IOP, ERG, and RNFL thickness in cat eyes will be measured. Moreover, hematoxylin and eosin (HE) staining and transmission electron microscope (TEM) will be examined to evaluate the protective function of BN52021 on retina. This study might provide new insights for the management of ocular blunt trauma, harmful to the retina.

\section{Methods}

\section{Cats and model}

The study protocol was approved by the Ethics Committee of the 307th Hospital of Chinese People's Liberation Army (PLA), affiliated hospital of Military Medical Sciences, Beijing, China. Healthy cats without eye diseases were enrolled in this study. The right cornea surface of all cats was treated with $1 \%$ tetracaine (PLA General Hospital) for topical anesthesia. All cats were treated with lower limb general anesthetized with Su-Mian-Xin (Military Veterinary institute of Military Medical sciences, Changchun, China, lot number: 20041010) and Ketamine (Beijing DoubleCrane Pharmaceutical Co., Ltd,Lot number:20050202) mixture $(1: 1,0.15 \mathrm{~mL} / \mathrm{kg})$. Cat was fixed and the right eye was opened with an eye speculum (World Precision Instruments, Sarasota, Florida). A small piece of lens paper was taped on eyes to protect the right cornea. The stainless steel cylinder, weight $0.195 \mathrm{~kg}$, was freely felled from the top of a self-made experimental steel (diameter $2 \mathrm{~cm}$, length $55 \mathrm{~cm}$ ) with the final impulse of $0.64 \mathrm{~kg} \cdot \mathrm{m} / \mathrm{s}$ to contuse the right eye. After contusion, cats with clear refractive media eyes were selected for further study. Cats with the symptoms such as corneal edema, cataract, hyphema, and vitreous hemorrhage were removed from this study.

Totally, 60 cats (male or female, 6 months old and weighted $2.5-3.5 \mathrm{~kg}$ ) with contused eyes were enrolled in this study. These 60 cats were randomly assigned into 5 groups: Group A $(\mathrm{n}=12), \mathrm{B}(\mathrm{n}=12), \mathrm{C}(\mathrm{n}=12), \mathrm{D}(\mathrm{n}=12)$, and $E(n=12)$. Another 10 healthy cats without eye diseases were used as normal control $(\mathrm{N})$ group $(\mathrm{n}=10)$. Norfloxacin eye drops (Wuhan Wujing medical Co., Ltd. Wuhan, China) was used to prevent potential eye infections. Cats in Group A to E were administrated with $0.5 \mathrm{~mL}$ of normal saline (NS), dimethyl sulphoxide (DMSO, Sigma-Aldrich, Saint Louis, MO, USA), 0.2 g/L BN52021, $1 \mathrm{~g} / \mathrm{L}$ BN52021 and $5 \mathrm{~g} / \mathrm{L}$ BN52021 (Tocris Bioscience, Bristol, UK), respectively. Cats in control group were administrated with $0.5 \mathrm{~mL}$ of NS. The choice of BN52021's concentrations was based on the clinical experience and the body weight of cats [14].

\section{Observation}

At $4 \mathrm{~h}$ (or $0 \mathrm{~h}$ ), 1 day, 3 days and 7 days after modeling, three cats in each group were anesthetized for inspections. All inspections were performed under general anesthesia (Su-Mian-Xin and Ketamine mixture). After anesthesia, we performed IOP and ERG measurements immediately. Then the medications of NS, DMSO and BN52021 were retrobulbarily injected into the right eyes. OCT was performed one hour after medication administrations. Then the three cats were euthanized for HE staining and TEM detection. The other cats being left untreated in each group were anesthetized and only administrated with NS, DMSO or BN52021.

\section{lop}

Methods for IOP measurement had previously been described by Pemp et al. [15]. Tropicamide was used to dilate the pupil. Tetracaine eye drops were used for local anesthetics on corneal surface. Measurements of IOP were performed immediately after the anesthesia, with a slit lamp-mounted Goldmann applanation tonometer (Haag-Streit, Bern, Switzerland).

\section{ERG}

Methods for ERG measurement had previously been described by Pawlyk et al. [16]. Briefly, cats were darkadapted at constant temperature for $1 \mathrm{~h}$. Tropicamide was used to dilate the pupil and all cats were anesthetized. The observations were performed by one of the authors. Roddominated responses were elicited in dark with $10-\mu \mathrm{s}$ flashes of white light $\left(3.556 \times 10^{-2} \mathrm{~cd} * \mathrm{~s} / \mathrm{m}^{2}\right)$ presented in a Ganzfeld dome. Maximum analysis time was $250 \mathrm{~ms}$ with the same flashes presented at $1 \mathrm{~Hz}$. The retinal signals were band-pass filtered between 0.1 and $75 \mathrm{~Hz}$, amplified 5,000-fold and averaged (5 repetitions). The amplitudes and latency periods of a- and b-waves, as well as the oscillograms after dark adaptation were recorded.

\section{OCT}

Methods for OCT measurement had previously been described by Ko et al. [17], with a Zeiss-humphery OCT 3.0 (Zeiss Humphery Instruments, Dublin, California, USA). RNFL thickness in macula was quantified and averaged (5 repetitions at different point).

\section{HE staining}

After the measurements of IOP, ERG and OCT, three cats in each group were euthanized for HE staining and TEM detection at each time point. Methods for the HE had previously been described by Assaf et al. [18]. In brief, the optic nerve near the eyeball were cut, fixed in $4 \%$ glutaraldehyde for $48 \mathrm{~h}$, dehydrated with a graded series of ethanol ( $2 \mathrm{~h}$ for each grade), transparented in dimethylbenzene and embedded in paraffin. Sections 


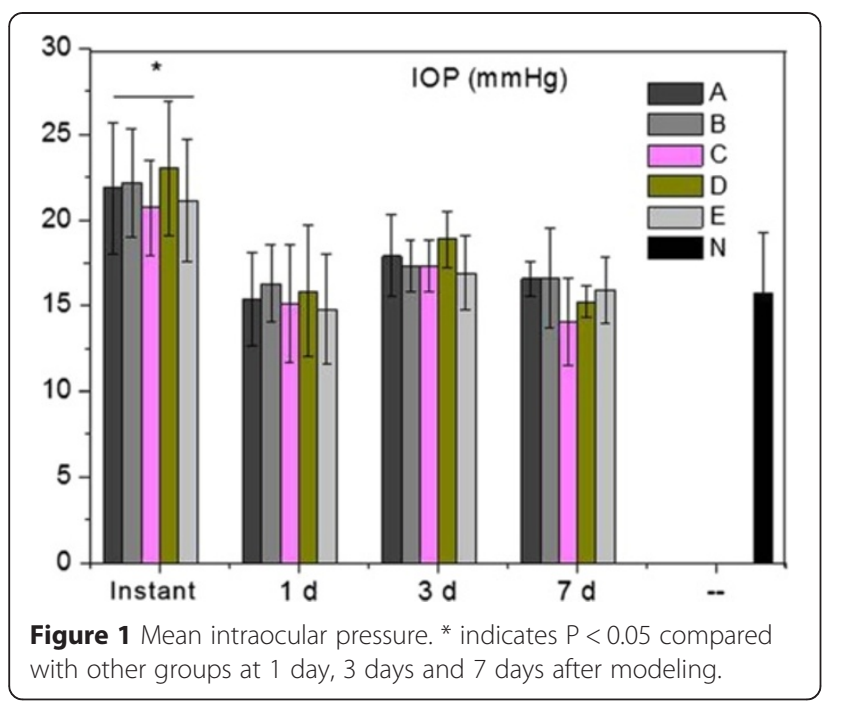

were cut by a microtome (Leica, Tokyo, Japan) and collected over polylysine treated slides. The slides were dewaxed, dehydrated, incubated with hematoxylin stain for $5 \mathrm{~min}$, differentiated with $1 \%$ hydrochloric acid alcohol for $30 \mathrm{~s}$, backed to blue with 1\% ammonium hydroxide and stained with $0.5 \%$ eosin solution for $5 \mathrm{~min}$. After the final dehydration and transparention, slides were sealed with neutralresinsize and examined using an optical microscope (Leica DMLS; Leica Microsystems Inc., Depew, New York, USA).

\section{TEM}

We performed the TEM detection according to the methods described by Wang et al. [19]. Three specimens in each group were cut into small pieces, $1 \mathrm{~mm} \times 1 \mathrm{~mm} \times$ $1 \mathrm{~mm}$. These pieces were washed with sodium cacodylate buffer, fixed in $2.5 \%$ glutaraldehyde, dehydrated with a

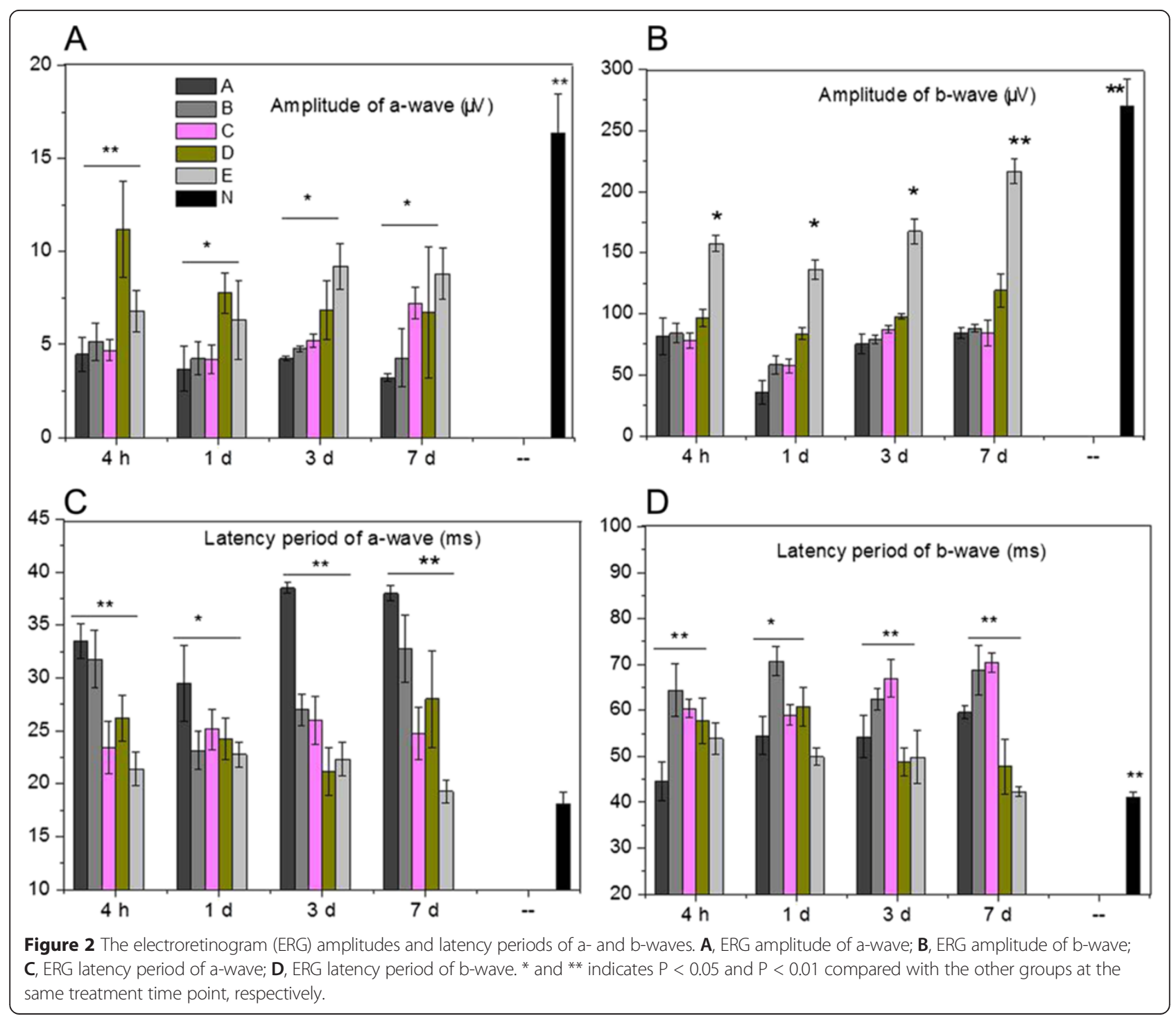


graded series of ethanol, sectioned with an ultramicrotome (Leica, Japan) and stained with saturated uranium acetate. TEM detections were performed by one of the authors using a JEM 100CX electron microscope (JEOL, Tokyo, Japan).

\section{Statistical analysis}

Each experiment was performed in triplicates, and the average values were calculated. SPSS 17.0 statistical software was used for data variance analysis. Experimental data were expressed as mean \pm standard deviation (SD). Unequal variances will be corrected with Welch. Comparison between two groups involved a paired sample t-test and comparison among three or more groups, a repeated measures one-way ANOVA and post hoc test. $\mathrm{P}<0.05$ was set as the threshold for significant difference.

\section{Results}

IOP

As shown in Figure 1, IOP levels at the onset after modeling were significantly higher than those at 1 day, 3 days and 7 days after modeling $(\mathrm{P}<0.05)$. The IOP levels in experimental groups were significantly decreased at 1 day, 3 days and 7 days after modeling. However, no significant difference was observed in IOP levels among groups at the same experimental point (Figure 1).

\section{ERG}

Comparing with Group N, the amplitudes of a- and bwaves in experimental groups were significantly decreased $(\mathrm{P}<0.05$, Figure $2 \mathrm{~A}, \mathrm{~B})$. The amplitude of a-wave in Group $A$ was lower than those in other groups $(P>0.05)$. The amplitude of a-wave in Group D showed a significant

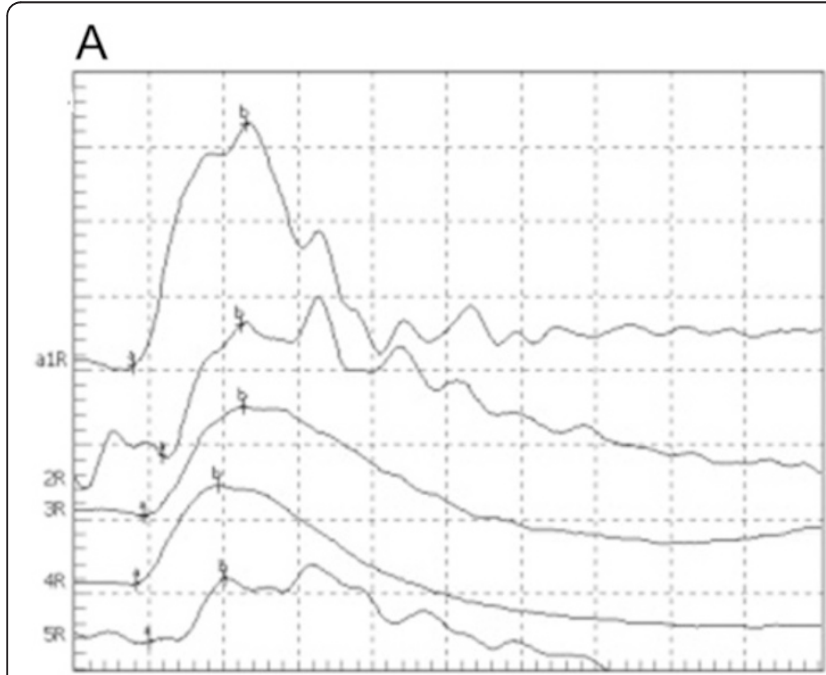

\section{B}

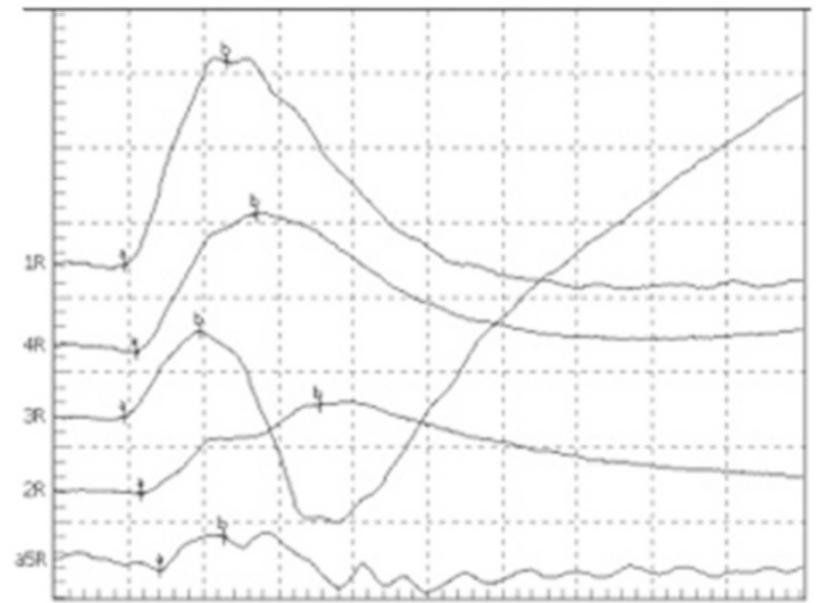

C

D
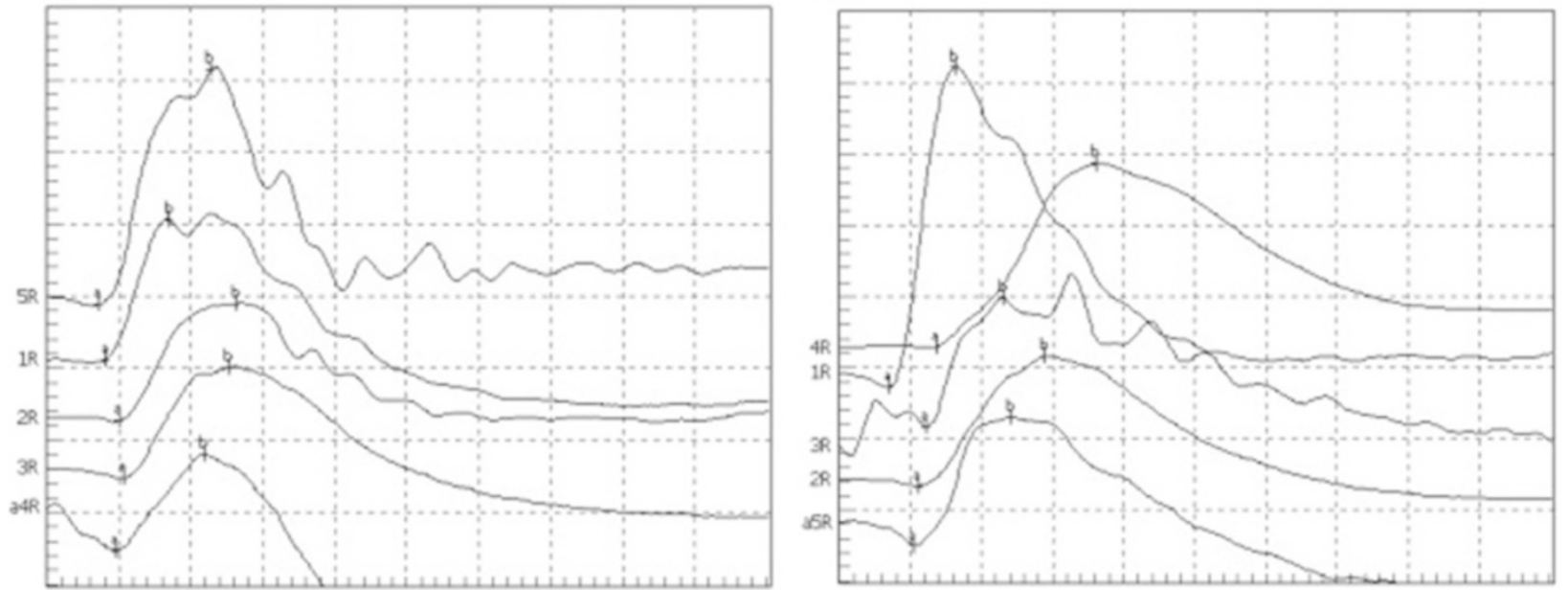

Figure 3 Tracings of oscillograhic records of cat retinas after dark adaptation. A - D, represent 4 inspection time points: 4 h, 1 day, 3 days and 7 days after modeling. From up to down, oscillograms for cats in Group E, D, C, B, and A, respectively. The stimulus duration is $250 \mathrm{~ms}$. 
decrease during the experimental period (Figure 2A). However, the amplitudes of a-wave in Group $\mathrm{E}$ at 3 days and 7 days after modeling were significantly higher than those in other groups $(\mathrm{P}<0.05$, Figure $2 \mathrm{~B})$. Moreover, the amplitude of b-wave in Group $\mathrm{E}$ was higher than those in other groups $(\mathrm{P}<0.0 .5)$.

The cats in Group A had significant longer latency periods of a-wave than other groups $(\mathrm{P}<0.01$, Figure $2 \mathrm{C})$. The latency period of b-wave in Group $\mathrm{E}$ was shorter than those in other groups $(\mathrm{P}<0.05$, Figure 2D). Moreover, the administration of BN52021 significantly decreased the latency period of $\mathrm{a}$ - and $\mathrm{b}$-waves in Group $\mathrm{E}(\mathrm{P}<0.05)$.
For the oscillograms measurement, the cats in Group E showed highest levels along with the experimental duration. Oscillograms of b-wave in Group E showed an obvious increment than those in other groups (Figure 3).

\section{OCT}

After modeling, RNFL thickness in Group A was significantly higher than that in control (Figure 4A-C). DMSO administration slightly decreased RNFL thickness (Figure 4D,E). Moreover, RNFL thickness decreased along with the increment of BN52021 concentration and handling time (Figure 4E-I). As shown in Figure 4 and

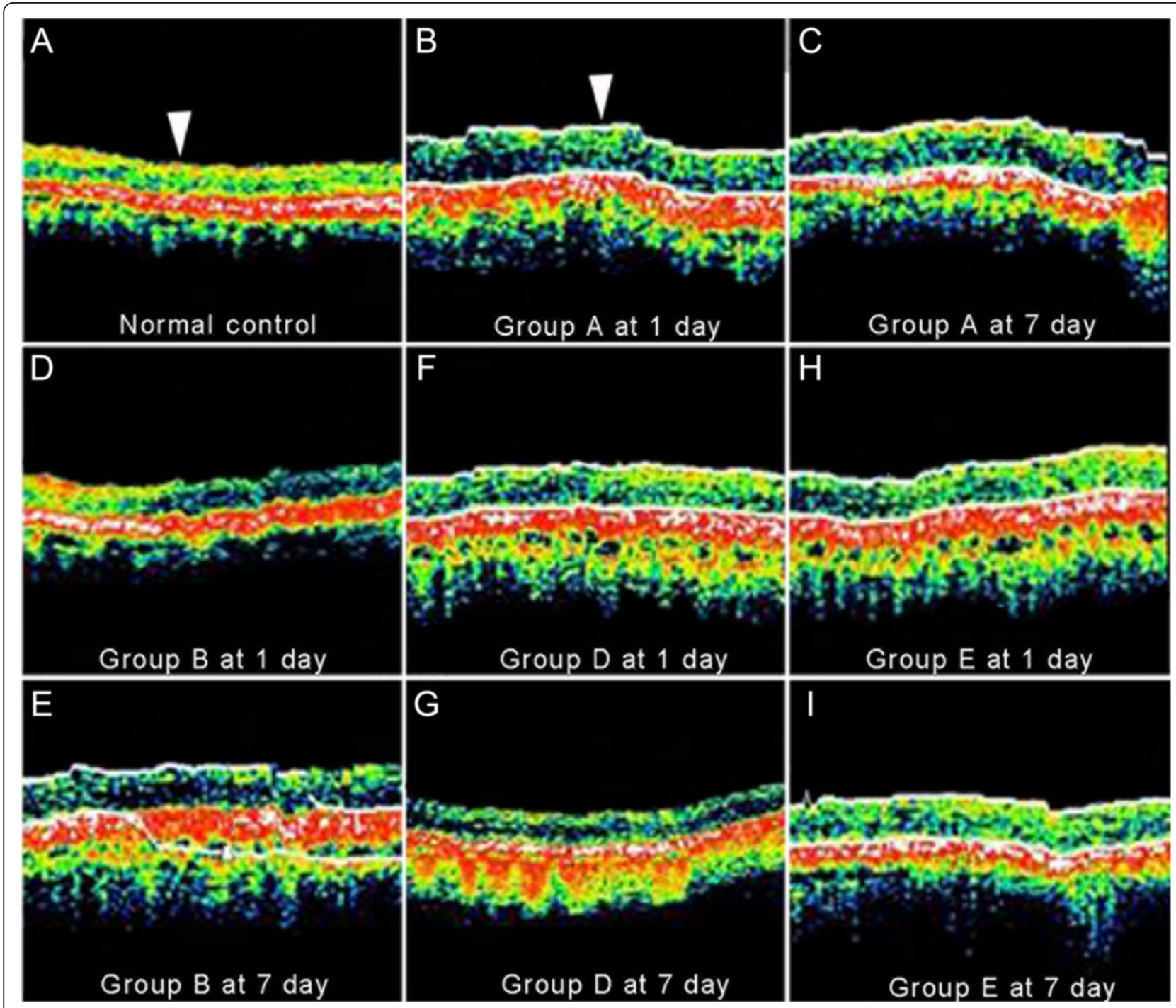

Figure 4 Optical coherence tomography (OCT) results of the retinal nerve fiber layer (RNFL) during the experimental periods. $\mathbf{A}$, OCT result of the normal RNFL; $\mathbf{B}$ and $\mathbf{C}$, OCT results of the RNFL in Group A at 1 day and 7 days after modeling with $0.5 \mathrm{~mL}$ of normal saline administration. An obvious swelling was observed in RNFL; $\mathbf{D}$ and $\mathbf{E}$, OCT results of the RNFL in Group B at 1 day and 7 days after modeling, with $0.5 \mathrm{~mL}$ of DMSO administration; $\mathbf{F}$ and $\mathbf{G}$, OCT results of the RNFL in Group D at 1 day and 7 days after modeling, with $0.5 \mathrm{~mL}$ of $1 \mathrm{~g} / \mathrm{L}$ BN52021 administration; $\mathbf{H}$ and $\mathbf{I}$, OCT results of the RNFL in Group $E$ at 1 day and 7 days after modeling, with $0.5 \mathrm{~mL}$ of $5 \mathrm{~g} / \mathrm{L}$ BN52021 administration. Comparing with the normal RNFL, all RNFLs in the experimental groups were swelling to some extent. For $\mathbf{A}, \mathbf{D}$ and $\mathbf{G}, \mathrm{bar}=150 \mu \mathrm{m}$, for $\mathbf{B}, \mathbf{C}, \mathbf{E}, \mathbf{F}, \mathbf{H}$ and $\mathbf{I}$, bar $=175 \mu \mathrm{m}$. 
Figure 5, RNFL thicknesses in Group D and E were significantly decreased than those in other groups $(\mathrm{P}<0.05)$. RNFL thickness in Group $\mathrm{E}$ at 7 days after modeling was at the same level as that in control (Figure 5).

\section{HE staining}

As shown in Figure 6, all cell layers of retina in control were clear and neatly arranged (Figure 6A). Cells were disorganized after modeling. Obvious swellings in RNFL and nucleus disintegration in ganglionic layers were observed (Figure 6B). RNFL swelling was slightly reduced by DMSO administration (Figure $6 \mathrm{C}$ ). The administration of high concentration of BN52021 significantly attenuated RNFL swelling. Ganglionic layers were neatly arranged in Group E (Figure 6D). Moreover, the anterior optic nerves were not damaged by contusions (Figure $6 \mathrm{E}-\mathrm{G}$ ). The nerve fibers were neatly arranged without difference between control and Group A (data in Group B-E were not shown).

\section{TEM}

Figure 7 showed the TEM results of the anterior optic nerves. Myelin sheath layers were neatly and tightly arranged in control cats (Figure 7A). Axons were degenerated and filled with vacuoles, and onion skin lesions were observed after modeling (Figure 7B). After being administrated with high concentration of BN52021, the lesions in Group E were significantly reduced and the spaces between axons were reduced (Figure 7C).

\section{Discussion}

Thierry et al. have reported that the presence of tritiated PAF $\left(\left[{ }^{3} \mathrm{H}\right] \mathrm{PAF}\right)$ specific binding sites which possess a $\mathrm{Kd}$

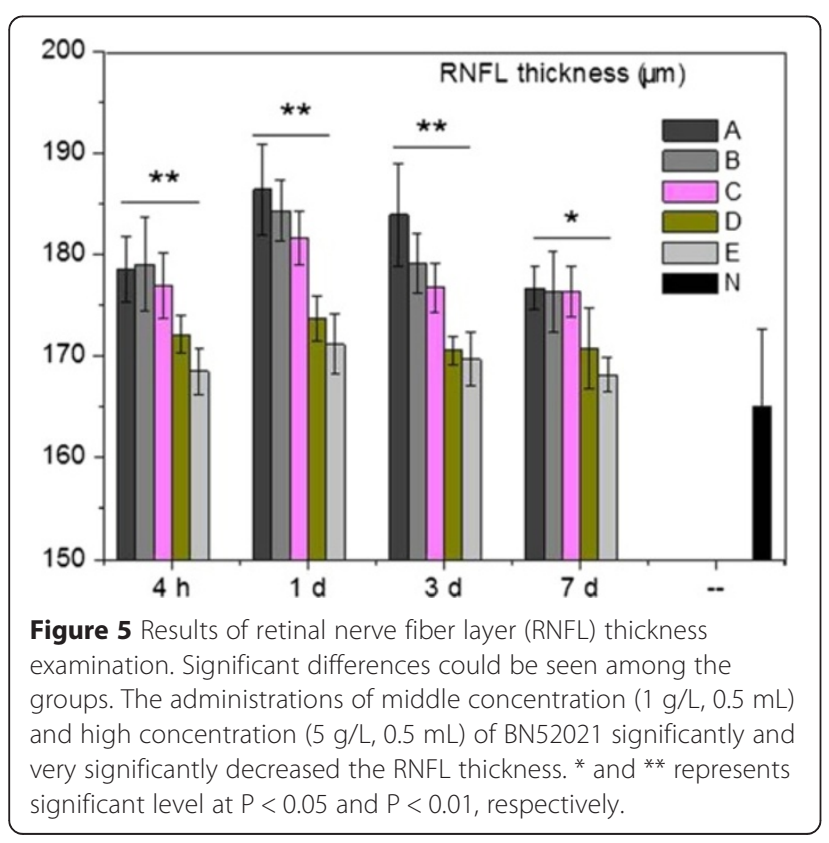

of $2.9 \pm 0.4 \mathrm{nM}$ and Bmax of $0.85 \pm 0.16 \mathrm{pmol} / \mathrm{mg}$ protein in membrane of albino rats retina. The binding of $\left[{ }^{3} \mathrm{H}\right] \mathrm{PAF}$ to these specific sites are saturable, specific, time-dependent and reversible and are considered to be related to PAF-induced disturbances of ERG b-wave [20].

As previously reported, BN52021's protective effect on retinal is dose-dependent. High concentration of BN52021 $\left(2 \times 10^{-5} \mathrm{M}\right)$ could partially inhibit the irreversible decrease of ERG b-wave amplitude induced by PAF-acether [9]. Our findings suggest that high concentration of BN52021 $(\geq 1 \mathrm{~g} / \mathrm{L})$ could partially restore the destroyed cat retina by decreasing the ERG amplitudes of a- and b-waves. This result is inconsistent with previous data showing that BN52021 administration could induce the inhibition of b-wave amplitude $[9,11]$. These results demonstrate again that BN52021 could partially inhibit the irreversible decrease of the ERG b-wave amplitude induced by PAFacether $[9,11,12]$. Moreover, the oscillograms can be considered to be reflection of retinal function [21]. In this study, the b-wave oscillograms in the group treated with high concentration of BN52021 showed obvious increment comparing with those in other groups treated with lower concentration of BN52021. These results suggest the retina as well as hyperpolarizing cone bipolar cells or horizontal cells might have been harmed by contusion. Retinal function might have been partially restored by BN52021 administrations.

Retinopathy is usually caused by injury and inflammatory on eyes, so did brain diseases as head trauma $[22,23]$. Inflammation serves as an important factor for the development of retinopathy [24]. Diseases as diabetes cause metabolic and physiologic abnormalities in retina, including the upregulation of iNOS, NF-kB and the increment of leukostasis and PAF. However, BN52021 could attenuate tissue damage caused by these abnormalities [10], including suppressing PAF induced paw swelling [25].

Evidences have suggested that BN52021 not only act as a PAF antagonist but also serve as a free radical scavenger for reactive oxygen species [26,27]. Thus, BN52021 could inhibit oxidation and decomposition of low-density lipoprotein [28]. BN52021 inhibits the increase of IOP and the damage to retina [29-32]. In this current study, administration of BN52021 reduced IOP levels and RNFL thickness, as well as attenuated the damage to RNFL. These results demonstrat that BN52021's protective effect on retinal function might relate to those inflammatory responses.

Nonetheless, there are also some limitations existing in this study. It might be more serious to perform the experiment with smaller grades of BN52021 concentration. It is more helpful to perform experiments on inflammation factors for investigation of BN52021's protective effect. In our study, the ERG amplitudes of a- and b-waves reduced 


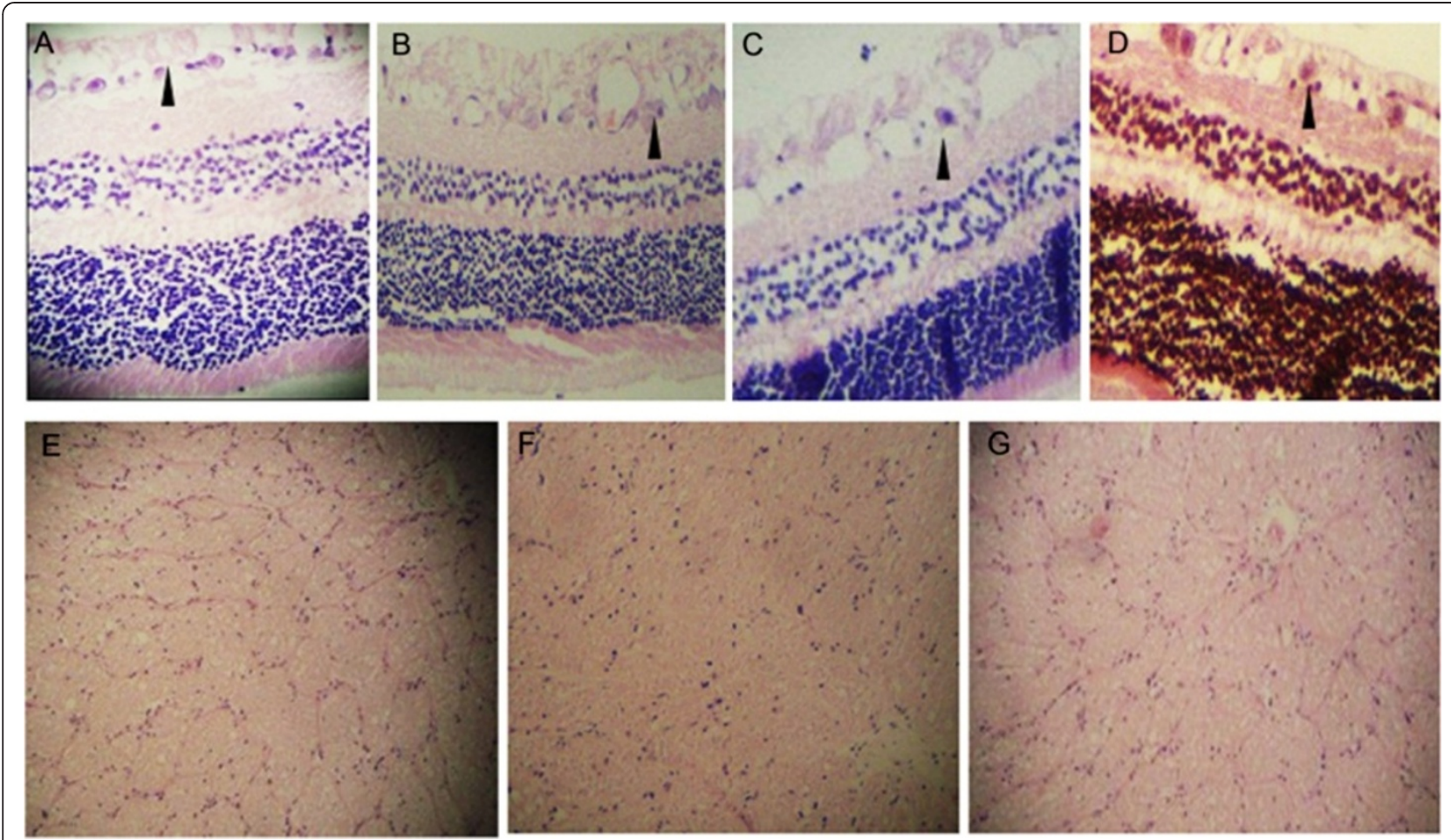

Figure 6 Hematoxylin and eosin (HE) staining results of the cell layers in retina and anterior optic nerves. $\mathbf{A}$, HE staining result of the cell layers in normal retina; $\mathbf{B}$ and $\mathbf{C}$, HE staining results of retina in Group A and B administrated with normal saline and DMSO at 1 day after modeling, respectively; D, HE staining result of retina in Group E administrated with high concentration ( $5 \mathrm{~g} / \mathrm{L}, 0.5 \mathrm{~mL}$ ) of BN52021 at 7 days after modeling. $\mathbf{E}$ to $\mathbf{G}$, HE staining results of anterior optic nerves in normal retina $(\mathbf{E})$ and contusion retina at $4 \mathrm{~h}(\mathbf{F})$ and 7 days $(\mathbf{G})$ after modeling. The arrows in Figure $\mathbf{A}-\mathbf{D}$ indicate the ganglionic layers in the RNFL. $\mathbf{A}$ to $\mathbf{D}$, original magnification $\times 400$. $\mathbf{E}$ to $\mathbf{G}$, original magnification $\times 100$.

by contusion were triggered by BN52021 administration. RNFL swelling and thickness increment were attenuated by BN52021 administration. Moreover, BN52021 administration significantly reduced the lesions in degenerated axons of anterior optic nerves. These suggested that high concentration of BN52021 ( $\geq 1 \mathrm{~g} / \mathrm{L})$ might have significant protective effects on retinal function of cat eyes. These hypotheses would be proved by more than one experiment on the inflammatory function of BN52021.

\section{Conclusions}

In conclusion, high concentration of BN52021 administration $(\geq 1 \mathrm{~g} / \mathrm{L})$ could significantly increase amplitudes of aand b-waves and reduce latency periods of a- and b- waves which were influenced by contusion. Moreover, BN52021 could decrease the RNFL thickness increased by contusion. This study demonstrates that BN52021 is a protective drug for retina function in cat eyes and might provide us with new insights for the management of ocular blunt trauma.

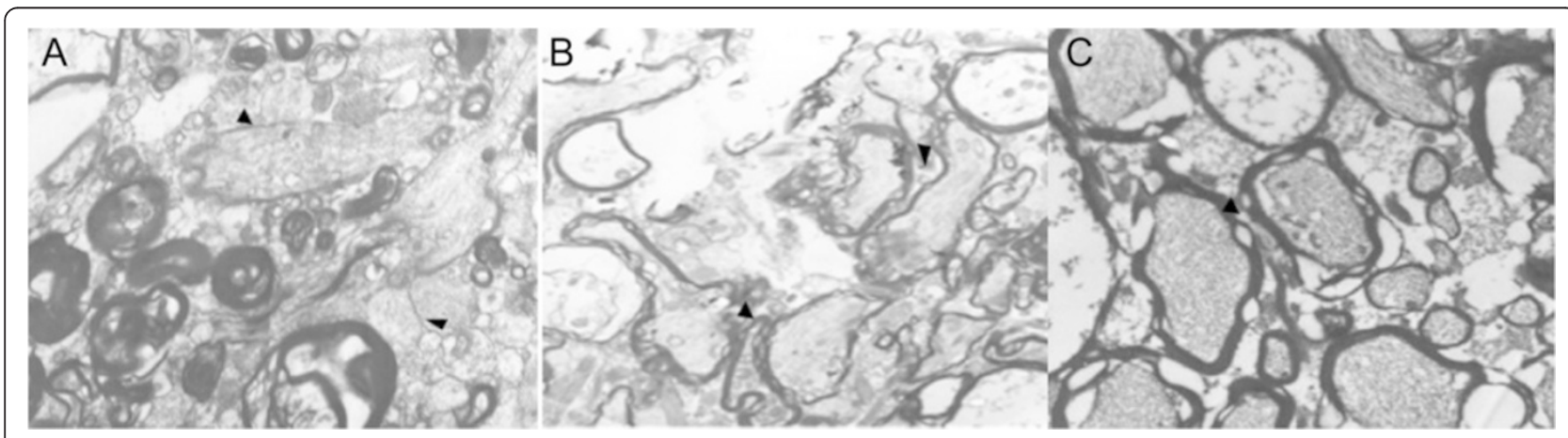

Figure 7 Transmission electron microscope (TEM) results of the anterior optic nerves in cat eyes. A, anterior optic nerves in normal cat eyes; $\mathbf{B}$, anterior optic nerves in contused cat eyes; C, anterior optic nerves in cat eyes administrated with high concentration $(5 \mathrm{~g} / \mathrm{L}, 0.5 \mathrm{~mL})$ of BN52021. Black arrows indicate the space between axons. A, original magnification $\times 10000$. B and C, original magnification $\times 4000$. 


\section{Abbreviations}

ERG: Electroretinogram; HE: Hematoxylin and eosin; IOP: Intraocular pressure; OCT: Optical coherence tomography; PAF: Platelet activating factor;

PLA: People's Liberation Army; RNFL: Retinal nerve fiber layer;

TEM: Transmission electron microscope.

\section{Competing interests}

The authors declare that they have no competing interests.

\section{Authors' contributions}

JFH participated in the design and writing of this study, HPZ provided technical support and participated in the study. ZJW and YFY are responsible for statistical analysis and participate in the study. $\mathrm{HMH}$ collected important background information. YY participated in the design and helped to draft the manuscript. All authors read and approved the final manuscript.

\section{Highlights}

1. No significant difference was observed in IOP level among groups.

2. BN52021 significantly increased rod ERG a- and b-wave amplitudes $(P<0.05)$.

3. RNFL thickness was decreased by high concentration of BN52021( $\geq 1 \mathrm{~g} / \mathrm{L})$.

4. Axons lesions induced by contusion were reduced after BN52021 administration.

\section{Author details}

'Department of Ophthalmology, 307 Hospital, PLA, Beijing 100071, China. ${ }^{2}$ Department of Ophthalmology, CHINA-JAPAN Friendship Hospital, No.2 Yinghua Dongjie, Hepingli, Beijing 100029, China. ${ }^{3}$ Department of Ophthalmology, The General Hospital of PLA, No. 28 Fuxing Road, Beijing 100853, China.

\section{Received: 7 December 2014 Accepted: 21 April 2015} Published online: 09 May 2015

\section{References}

1. Samuel MA, Tawansy KA. Pediatric Retinal Trauma. In: Pediatric Retina. Berlin Heidelberg: Springer; 2011. p. 423-31.

2. Armstrong GW, Kim JG, Linakis JG, Mello MJ, Greenberg PB. Pediatric eye injuries presenting to United States emergency departments: 2001-2007. Graefes Arch Clin Exp Ophthalmol. 2013;251(3):629-36.

3. Hirashima T, Kita M, Yoshitake S, Hirose M, Oh H. Massive vitreous gel incarceration into the subretinal space following traumatic retinal detachment in a young patient: a case report. Clin Ophthalmol. 2011;5:1539-41.

4. Acar U, Yildiz EH, ACAR DE, Altiparmak UE, Akkaya ZY, Burcu A, et al. Posttraumatic intraocular pressure elevation and associated factors in patients with zone I open globe injuries. Ulus Travma Acil Cerrahi Derg. 2013;19(2):115-8.

5. Turalba AV, Shah AS, Andreoli MT, Andreoli CM, Rhee DJ. Predictors and outcomes of ocular hypertension after open-globe injury. J Glaucoma. 2014;23(1):5-10.

6. Nakayama Y, Yokoi T, Sachiko N, Okuyama M, Azuma N. Electroretinography combined with spectral domain optical coherence tomography to detect retinal damage in shaken baby syndrome. J AAPOS. 2013;17(4):411-3.

7. Ahn HB, Hur MC, Lee JW. Analysis of the peripapillary retinal nerve fibre layer thickness using optical coherence tomography in traumatic optic neuropathy. Neuroophthalmology. 2012;36(2):42-7.

8. Schuman JS, Hee MR, Puliafito CA, Wong C, Pedut-Kloizman T, Lin CP, et al. Quantification of nerve fiber layer thickness in normal and glaucomatous eyes using optical coherence tomography: a pilot study. Arch Ophthalmol. 1995;113(5):586-96.

9. Doly M, Bonhomme B, Braquet P, Chabrier P, Meyniel G. Effects of plateletactivating factor on electrophysiology of isolated retinas and their inhibition by BN 52021, a specific PAF-acether receptor antagonist. Immunopharmacology. 1987;13(3):189-94.

10. Xia S-H, Hu C-X, Zhao Z-L, Xia G-D, Di Y. Significance of platelet activating factor receptor expression in pancreatic tissues of rats with severe acute pancreatitis and effects of BN52021. World J Gastroenterol. 2007;13(21):2992-8.

11. Nitoda E, Moschos MM, Mavragani CP, Koutsilieris M. Ocular actions of platelet-activating factor: clinical implications. Expert Opin Ther Targets. 2012;16(10):1027-39.
12. Panetta $T$, Marcheselli VL, Braquet $P$, Spinnewyn B, Bazan NG. Effects of a platelet activating factor antagonist (BN 52021) on free fatty acids, diacylglycerols, polyphosphoinositides and blood flow in the gerbil brain: inhibition of ischemia-reperfusion induced cerebral injury. Biochem Biophys Res Commun. 1987;149(2):580-7.

13. Einhäuser W, Moeller GU, Schumann F, Conradt J, Vockeroth J, Bartl K, et al. Eye-head coordination during free exploration in human and cat. Ann N Y Acad Sci. 2009;1164(1):353-66.

14. Xia S. Role and mechanism of platelet activating factor receptor antagonist (BN52021) in early stage of severe acute pancreatitis in rats. Third Military Medical University 2009.

15. Pemp B, Polska E, Karl K, Lasta M, Minichmayr A, Garhofer G, et al. Effects of antioxidants (AREDS medication) on ocular blood flow and endothelial function in an endotoxin-induced model of oxidative stress in humans. Invest Ophthalmol Vis Sci. 2010;51(1):2-6.

16. Pawlyk BS, Bulgakov OV, Liu X, Xu X, Adamian M, Sun X, et al. Replacement gene therapy with a human RPGRIP1 sequence slows photoreceptor degeneration in a murine model of Leber congenital amaurosis. Hum Gene Ther. 2010;21(8):993-1004.

17. Ko TH, Fujimoto JG, Schuman JS, Paunescu LA, Kowalevicz AM, Hartl I, et al. Comparison of ultrahigh-and standard-resolution optical coherence tomography for imaging macular pathology. Ophthalmology. 2005;112(11):1922. e1921-15.

18. Assaf Y, Blumenfeld-Katzir T, Yovel Y, Basser PJ. AxCaliber: a method for measuring axon diameter distribution from diffusion MRI. Magn Reson Med. 2008;59(6):1347-54.

19. Wang $X-Y$, Vannucchi M-G, Nieuwmeyer F, Ye J, Faussone-Pellegrini M-S, Huizinga JD. Changes in interstitial cells of Cajal at the deep muscular plexus are associated with loss of distention-induced burst-type muscle activity in mice infected by Trichinella spiralis. Am J Pathol. 2005;167(2):437-53.

20. Thierry A, Doly M, Braquet P, Cluzel J, Meyniel G. Presence of specific platelet-activating factor binding sites in the rat retina. Eur J Pharmacol. 1989;163(1):97-101.

21. Marmor MF, Zrenner E. Standard for clinical electroretinography (1994 update). Doc Ophthalmol. 1995;89(3):199-210.

22. Adamis A. Is diabetic retinopathy an inflammatory disease? $\mathrm{Br} J$ Ophthalmol. 2002;86(4):363-5.

23. Shah GK, Penne R, Grand MG. Purtscher's retinopathy secondary to airbag injury. Retina. 2001;21(1):68-9.

24. Kern TS. Contributions of inflammatory processes to the development of the early stages of diabetic retinopathy. J Diabetes Res. 2006;2007:95103. 1-14.

25. Wang J-P, Teng C-M. Effects of anti-inflammatory drugs on rat hind-paw swelling caused by phospholipase A2 from Naja naja atra venom. Naunyn Schmiedebergs Arch Pharmacol. 1991;344(3):377-81.

26. Shi H, Niki E. Stoichiometric and kinetic studies on Ginkgo biloba extract and related antioxidants. Lipids. 1998:33(4):365-70.

27. Kaplan M, Mutlu EA, Benson M, Fields JZ, Banan A, Keshavarzian A. Use of herbal preparations in the treatment of oxidant-mediated inflammatory disorders. Complement Ther Med. 2007;15(3):207-16.

28. de Assis EF, Silva AR, Caiado LF, Marathe GK, Zimmerman GA, Prescott SM, et al. Synergism between platelet-activating factor-like phospholipids and peroxisome proliferator-activated receptor $\gamma$ agonists generated during low density lipoprotein oxidation that induces lipid body formation in leukocytes. J Immunol. 2003;171(4):2090-8.

29. Jager $G$, Van Delft J, Van Haeringen N, Verbeij N, Braquet P. Antagonist of platelet-activating factor prevents prostaglandin E2 induced ocular hypertension in rabbits. Prostaglandins. 1993;45(1):97-105.

30. Dineley K, Richards L, Votyakova T, Reynolds I. Zinc causes loss of membrane potential and alters production of reactive oxygen species in isolated brain mitochondria. J Neurochem. 2002;81(s1):101-5.

31. Remé C, Wei Q, Munz K, Jung H, Doly M, Droy-Lefaix M-T. Light and lithium effects in the rat retina: modification by the PAF antagonist BN 52021. Graefes Arch Clin Exp. 1992;230(6):580-8.

32. Menerath J, Cluzel J, Droy-Lefaix MT, Doly M. Experimental electroretinographic exploration of retinal ischemia: preventive use of free radical scavengers and anti-PAF agents. J Ocul Pharmacol Ther. 1997;13(1):81-8. 